\title{
IDENTIFICACIÓN DE INDICADORES PROPIOS DE ESTUDIANTES DE TALENTO MATEMÁTICO: FLUIDEZ, FLEXIBILIDAD, ORIGINALIDAD, ELABORACIÓN Y CREATIVIDAD
}

\author{
Óscar Roldán Blay \\ Irene Ferrando Palomares \\ Universitat de València
}

\begin{abstract}
RESUMEN: En el presente trabajo se describen los resultados de un estudio exploratorio cuyo objetivo es identificar características de estudiantes de talento matemático cuando se enfrentan a una secuencia de problemas de solución múltiple. Para abordar este propósito, se han seleccionado dos muestras de alumnos: una formada por estudiantes de entre 15 y 17 años participantes en un programa dirigido a estudiantes con talento matemático, y la otra formada por alumnos con una sólida formación matemática. A continuación, se ha diseñado una secuencia de cuatro tareas de resolución múltiple y se ha realizado un análisis cualitativo de las respuestas con el objeto de identificar componentes cognitivos diferenciadores de los estudiantes de talento matemático respecto a estudiantes con una formación matemática sólida en base a diferentes indicadores: fluidez, flexibilidad, originalidad, elaboración y creatividad.
\end{abstract}

PALABRAS CLAVE: talento matemático, flexibilidad, creatividad, tareas de solución múltiple.

\section{IDENTIFICATION OF SPECIFIC INDICATORS OF MATHEMATICAL TALENT: FLUENCY, FLEXIBILITY, ORIGINALITY, ELABORATION AND CREATIVITY}

\footnotetext{
ABSTRACT: This paper describes the results of an exploratory study seeking to identify characteristics of mathematically talented students when faced with a sequence of multiple-solution problems. With this aim, two samples of students have been selected: one formed by students between 15 and 17 years old participating in a programme addressed to mathematically talented students, and the other formed by students with a solid mathematical background. A sequence of four multiple-choice tasks was then designed and
} 
a qualitative analysis of the answers was carried out in order to characterise the profile of the mathematically talented students based on different indicators: flexibility, originality, elaboration and creativity.

KEYWORDS: Mathematical talent, flexibility, creativity, multiple solution tasks.

Recibido: 28/01/2021

Aceptado: 11/03/2021

Correspondencia: Óscar Roldán Blay, Facultad de Matemáticas, Universitat de València, C/ Dr. Moliner, 50, 46100 Burjassot, Valencia. Email: oscar.roldan@uv.es

\section{INTRODUCCIÓN Y OBJETIVOS}

En los últimos años hay un interés creciente centrado en la problemática de detectar y atender a los alumnos superdotados y de talento. Sin embargo, la dificultad para el profesorado no radica solo en la atención a los alumnos de alta capacidad matemática, sino en la detección; en efecto, para los docentes es difícil encontrar documentos que informen sobre las características cognitivas de estos estudiantes. Esto puede causar que estudiantes muy capaces y con ganas de aprender caigan en la frustración derivada de un sistema educativo que tiende a homogeneizar al conjunto de los alumnos.

Además, hay una confusión entre alumnos de alta capacidad matemática (en adelante, alumnos de talento matemático) y alumnos superdotados. Los estudiantes son identificados como superdotados si tienen un cociente intelectual mayor o igual que 130 y a menudo este criterio deja sin derecho a atención diferenciada a muchos estudiantes que poseen altas capacidades intelectuales específicas en algún área académica pero no en otras, en particular a los estudiantes de talento matemático. En el ámbito de la psicometría existen diversos cuestionarios estandarizados para identificar la superdotación que se utilizan para identificar a los estudiantes con altas capacidades (por ejemplo, los tests de Raven y de Thurstone, el test de pensamiento creativo de Torrance), así como otros más específicos centrados en las matemáticas como el test de diagnóstico matemático de Stanford. En el contexto de las matemáticas, varios estudios han mostrado que los métodos mencionados no tienen suficiente fiabilidad (Miller, 1990) y que se obtienen mejores resultados utilizando baterías cuidadosamente diseñadas de problemas de matemáticas (Díaz et al., 2008).

Jaime y Gutiérrez (2014) exponen una extensa lista de características que suelen presentar los alumnos de talento matemático, entre las se pueden destacar: formular preguntas más allá de la tarea, flexibilidad, originalidad, reconocimiento de patrones y relaciones, estrategias eficientes, simplificación de procesos, rapidez para aprender y resolver problemas, generalización y transferencia. En particular, según estos autores, los alumnos de talento matemático destacan por su rapidez y su originalidad a la hora de resolver problemas. Otro aspecto importante a observar 
en los estudiantes de talento matemático es la creatividad. Leikin (2013) desarrolla una investigación centrada en diseñar y evaluar un modelo para evaluar la creatividad matemática que parte de la premisa de que la creatividad es la conjunción de diferentes componentes que, según Torrance (1974), son: fluidez, flexibilidad, originalidad y elaboración.

Con el objeto de identificar características diferenciadoras de los estudiantes de talento matemático en relación a estudiantes con una sólida formación matemática, se ha realizado un estudio exploratorio comparativo en el que se analizan las producciones de ambos tipos de estudiantes. A través de este estudio se pretenden abordar los siguientes objetivos:

- Diseñar de una secuencia de tareas de solución múltiple (MST en adelante, acrónimo del inglés Multiple Solution Task) y analizar cualitativamente las respuestas a la secuencia de tareas.

- Identificar características diferenciadoras en las producciones de estudiantes de talento matemático.

- Obtener una categorización de indicadores de talento matemático.

\section{MARCo teórico}

En este apartado se tratarán dos aspectos que fundamentan el desarrollo de esta investigación: por un lado, se argumentará y explicará cómo utilizar secuencias de problemas en la identificación de estudiantes de talento matemático, y en particular se describirán las MST como herramienta útil de detección; a continuación se describirán algunos trabajos centrados en caracterizar el perfil de los estudiantes de talento matemático.

\subsection{La resolución de problemas en la identificación de estudiantes de talento matemático}

Al tratar el concepto de "problema" es conveniente tener en cuenta la distinción hecha por Polya (1965) entre "problemas de rutina" (habitualmente Ilamados "ejercicios") y "problemas por resolver" o "problemas por demostrar" (genéricamente, "problemas"). Los ejercicios son tareas que el resolutor ya sabe resolver, porque son idénticas a otras resueltas previamente o porque consisten en aplicar de manera directa conocimientos previos. Por el contrario, los problemas son tareas que, en principio, el resolutor no sabe resolver y le obligan a explorar vías de trabajo que nunca antes había seguido.

Según se recoge en numerosos trabajos del ámbito de la educación matemática, la mejor forma de observar los procesos de razonamiento de los estudiantes de matemáticas es a través de la resolución de problemas. De hecho, pese a la escasez de investigaciones sobre estudiantes de talento matemático publicadas, la mayoría de ellas están relacionadas con la resolución de problemas (Jaime y Gutiérrez, 2017).

En investigaciones recientes (Leikin y Lev, 2007 y Leikin, 2011), se han utilizado las MST como herramienta metodológica para analizar el proceso de resolución de problemas. Una MST es una tarea en la cual se pide al alumno explícitamente resol- 
ver un problema de matemáticas de diferentes formas. Dos soluciones se consideran diferentes si se verifica alguna de las siguientes condiciones:

a) Se basan en representaciones diferentes de algunos conceptos matemáticos involucrados en la tarea.

b) Se basan en propiedades (definiciones o teoremas) diferentes de objetos matemáticos de un mismo campo en particular.

c) Se basan en propiedades diferentes de un objeto matemático en campos distintos.

Al utilizar en el diseño de una investigación las MST, es necesario contar con un conjunto detallado de las soluciones posibles a dicho problema: esto es lo que Leikin y Lev (2007) Ilaman espacios de soluciones de MST. En el presente estudio, tal y como se describirá en el apartado de metodología, el diseño de la secuencia de problemas se basará en cuatro MST.

\subsection{Antecedentes sobre las características de los estudiantes de talento matemático}

En los últimos años se han desarrollado, en el ámbito de la educación matemática, diferentes investigaciones empíricas centradas en identificar rasgos de comportamiento de los estudiantes de talento matemático. Algunos estudios se basan en la observación de estudiantes de talento matemático durante el proceso de resolución de problemas: el primer referente es el trabajo de Krutetski (1976), donde se identifican como rasgos identificativos la rapidez y flexibilidad de razonamiento, memoria matemática, y habilidades de generalización, de manejo de conceptos abstractos, de identificación y uso de estructuras matemáticas (Jaime y Gutiérrez, 2017). Otros trabajos que siguen esta misma metodología se basan en observar a estudiantes de talento matemático resolver algún tipo concreto de problema y, sí, permiten identificar características específicas de determinadas áreas de las matemáticas (véase Jaime y Gutiérrez, 2014).

Otros estudios centrados en identificar características de los estudiantes de talento matemático se basan en una metodología de investigación comparativa: toman una muestra de estudiantes que previamente ya han sido identificados como talentosos y comparan sus formas de enfrentarse a la resolución de problemas frente a estudiantes con otros perfiles. Uno de los primeros referentes en relación a la caracterización de los estudiantes de talento matemático es el trabajo de Shapiro (1965) que apunta que, en dichos alumnos, el desarrollo de la generalización de resultados tras el proceso de resolución de un problema matemático se da a edades tempranas en este tipo de estudiantes. Davydov (1990) añade que la generalización es inseparable de las operaciones con lo abstracto y Krutetskii (1976), en base a un estudio que pretendía analizar y comparar la habilidad de generalizar en estudiantes tanto "normales" como talentosos, caracterizó tres tipos de razonamiento matemático en los alumnos cuyas características se resumen a continuación basándonos en la descripción de Callejo (1994). El tipo analítico, caracterizado por un predominio de la componente lógico-verbal y una componente visual-pictórica débil; este tipo de alumnos suele intentar enfrentarse a problemas de forma analítica incluso si el contexto sugiere plantearlos geométricamente. El tipo geométrico, caracterizado por 
una componente visual-pictórica más desarrollada que la lógico-verbal; este tipo de alumnos suele interpretar todo visualmente e intenta trabajar en base imágenes. El tipo armónico, capaz de usar tanto esquemas visuales como fórmulas abstractas y pasar de un modelo a otro; generalmente, a los alumnos de altas capacidades matemáticas se les asocia este tipo de razonamiento.

En las últimas décadas se han hecho más estudios con el fin de verificar y analizar la relación que existe entre el talento matemático y la habilidad de resolver problemas. Sriraman (2003) hizo un estudio que verifica que hay una relación entre el talento matemático, la habilidad en la resolución de problemas, y la habilidad para generalizar.

Jaime y Gutiérrez (2017), recogen una panorámica de los resultados de investigaciones sobre estudiantes de talento matemático y resumen así los resultados de algunos de estos estudios centrados en las características:

Una característica típica de la resolución de problemas por estudiantes con alta capacidad es la originalidad de sus procedimientos, pues con frecuencia siguen formas de resolución atípicas, en las que combinan los conocimientos matemáticos de manera sorprendente. Estos estudiantes suelen mostrar mucha intuición al resolver problemas, pues son capaces de llegar a la solución de un problema incluso aunque no dispongan de los conocimientos matemáticos necesarios para escribir una justificación detallada de la misma. También es reconocida como una característica típica de los estudiantes con alta capacidad matemática la rapidez para comprender nuevos conceptos, propiedades o procedimientos. (p. 77)

La originalidad a la que se refieren estos autores es, según Torrance (1974), una de las componentes de la creatividad. En efecto, según este autor (citado en Leikin, 2013), la creatividad puede entenderse como un constructo dividido en cuatro componentes:

- Fluidez: se refiere a la continuidad de ideas, flujo de asociaciones y uso de conocimiento básico y universal.

- Flexibilidad: está asociada con los cambios de ideas, con la capacidad de abordar problemas de más de una forma, obteniendo más de una resolución.

- Originalidad: se caracteriza por una forma particular de pensar que da lugar a productos de actividad mental o artística únicos. Según Leikin (2013), la originalidad es la componente principal de la creatividad.

- Elaboración: se refiere a la capacidad de crear, describir y generalizar ideas.

Leikin y Lev (2007) y Leikin (2013) abordan una investigación centrada en estudiar las características de los alumnos de talento matemático; en particular, identifican en base al análisis de las resoluciones de MST, características que se remitan a la noción de creatividad. En el presente estudio se aborda una adaptación a pequeña escala del trabajo de Leikin (2013) con el objetivo de identificar características diferenciadoras de los alumnos de talento matemático respecto a estudiantes con una sólida formación matemática. En el siguiente apartado se describen los aspectos metodológicos del estudio. 


\section{Metodología}

Este estudio pretende comparar dos grupos de estudiantes. A continuación, se presentan los detalles de las características de los participantes en el estudio.

\subsection{Descripción de los participantes}

El presente estudio tiene un carácter exploratorio, es por ello que se ha trabajado con una pequeña muestra de estudiantes. Se trata de una muestra de conveniencia formada por participantes que han decidido acceder de forma voluntaria a hacer la experiencia.

El primer grupo está compuesto por 5 estudiantes del Máster Universitario en Profesor/a de Secundaria (especialidad Matemáticas) de la Universitat de València. Se trata de personas que han finalizado con éxito sus estudios en los Grados de Matemáticas o Física, por tanto, tienen una formación matemática sólida. Sus edades respectivas son 22, 22, 22, 23 y 24 años. Si bien se trata de alumnos con una sólida formación matemática, ninguno ha recibido formación complementaria por altas capacidades ni se ha formado en resolución de problemas hasta este curso. El criterio para elegirlos ha sido la calificación obtenida en la materia dedicada a resolución de problemas del máster, se ha escogido a aquellos alumnos que han sacado las mejores notas.

El segundo grupo está formado por 6 alumnos que participan en el programa de estimulación del talento matemático ESTALMAT. Dos de los alumnos, de 15 y 16 años respectivamente, van a $4^{\circ}$ de ESO, y los otros 4 son alumnos de $1^{\circ}$ de Bachiller, y sus edades respectivas son 16, 16, 16 y 17 años. Para una información completa sobre el proyecto ESTALMAT, conviene remitirse a los trabajos de De Guzmán (s.f.) y Miralles (2008).

A continuación, se describirá el diseño de la secuencia de problemas que corresponden a las MST cuyas características se han comentado en el marco teórico.

\subsection{Secuencia de tareas}

Con el objeto de identificar características diferenciadoras de alumnos de talento matemático, se ha diseñado una secuencia de cuatro MST. Leikin (2011) expone tres ejemplos de actividades MST que son accesibles para la muestra de estudiantes seleccionados, por lo que se han traducido los enunciados y se han incluido en la secuencia utilizada en el presente trabajo. Además, se ha incluido un cuarto problema, propuesto por uno de los autores de este estudio, que también verifica las condiciones para ser considerado una MST. A continuación, se formulan los enunciados de cada uno de los problemas y se comentan las posibles soluciones del primero.

El primer problema es de geometría, y se enuncia como sigue.

Problema 1: Probar que, en cualquier triángulo rectángulo, la mediana que une el vértice del ángulo recto con el punto medio de la hipotenusa, mide igual que media hipotenusa.

Para que sea más fácil entender las ideas de las soluciones, que serán comentadas en el siguiente apartado, se establecen ciertas convenciones: el vértice del ángulo recto se llamará $A$, los otros dos, $B$ y $C$. El punto medio de la hipotenusa $\overline{B C}$ se Ila- 
mará M. La longitud de un lado del triángulo se llamará con la misma letra que su vértice opuesto, pero en minúscula, y la longitud del segmento que piden, $\overline{A M}$, se denotará $x$. En la Figura 1 se representa geométricamente el enunciado del problema.

Figura 1. Enunciado del problema 1

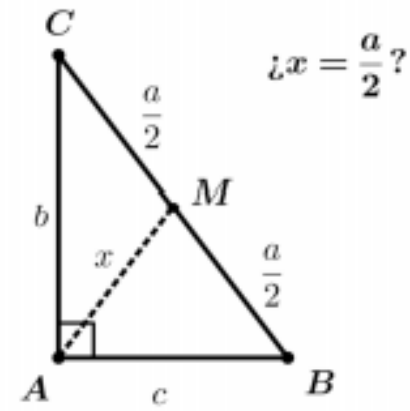

El segundo problema, si bien admite una resolución geométrica, tiene un enunciado algebraico.

Problema 2: Si a y $b$ son dos números que suman 1 , ¿qué relación hay entre $a^{2}+$ b y $a+b^{2}$ ?

El tercer problema propuesto es un problema de optimización basado en conceptos geométricos:

Problema 3: De todos los rectángulos con un mismo perímetro, ¿cuál es el que tiene la diagonal más pequeña?

El último problema, propuesto en una de las ediciones de las Olimpiadas Matemáticas de Educación Secundaria organizadas por la Societat d'Ensenyants de Matemàtiques de la Comunitat Valenciana (SEMCV), tiene una complejidad mayor a los tres previos y cumple las características buscadas en este estudio.

Problema 4: En un triángulo rectángulo, los catetos miden 5 y 12. ¿Cuánto mide el radio de la circunferencia inscrita?

Si bien el presente estudio realizado es pequeño y, por tanto, sus conclusiones son más bien orientativas y sus resultados, exploratorios, su dimensión permite analizar con mucho nivel de detalle una gran cantidad de factores: cuántas soluciones se ofrecen, en qué orden, cuál es la primera de todas que se les ocurrió, o cuáles son los valores de creatividad calculados según la fórmula que propone Leikin (2013). En los siguientes apartados se presentan los detalles del desarrollo de la experiencia y de los criterios de análisis de las producciones de los participantes.

\subsection{Desarrollo de la experiencia}

Dado que, por cuestiones ajenas a la investigación, no era posible reunir a los participantes para que hicieran la prueba simultáneamente, se fijaron unas instrucciones claras que todos los participantes se comprometieron a cumplir. Se limitó a 2 horas 
el tiempo para abordar los cuatro problemas, con el objeto de fijar unas condiciones comunes a todos que fueran suficientes para trabajar sobre cada problema de forma que se pudieran encontrar varias resoluciones para cada uno. Además, se indicó a los participantes que debían resolver los problemas de forma autónoma sin consultar ningún tipo de fuentes. Las instrucciones, tal y como se presentaron a los participantes, son las siguientes:

- Resuelve estos 4 problemas por tantos caminos distintos como se te ocurran.

- Tienes un total de 2 horas para hacerlo (30 minutos aprox. por pregunta), no dediques más que eso ni hagas ningún tipo de trampa.

- Al terminar, escanea tus soluciones y envíalas al correo XXXX.

- No hace falta ser excesivamente formal. Lo que más me importa es el procedimiento, la creatividad, más que si te has equivocado en alguna operación o algo así.

- Puedes usar calculadora y material de dibujo si quieres.

\subsection{Análisis}

En este apartado se presentará, en primer lugar, el sistema de organización de los datos, a continuación, se describirá, tomando como ejemplo el primer problema, cómo se han obtenido y organizado los espacios de soluciones de cada problema. Finalmente se detallarán los aspectos analizados en las resoluciones.

\subsubsection{Organización de los datos}

Una vez recopiladas las producciones de los participantes en el estudio, se procede a la fase de análisis cualitativo de las resoluciones. En primer lugar, se han codificado los datos.

A los alumnos se les asigna la letra A, seguida de una letra ( $M$ si es del Máster y $E$ si es de ESTALMAT) y un número del 1 al 11, de forma que los primeros 5 se corresponden con los alumnos del máster y los últimos 6 se corresponden con los estudiantes del proyecto ESTALMAT.

A los problemas se les asigna la letra $\mathrm{P}$ seguida del número del problema, numerados en el mismo orden en el que fueron presentados (P1, P2, P3 y P4). Cada solución se codifica con la letra $S$, seguida del número de problema, y una letra, en orden alfabético, de forma que las soluciones del problema 1, por ejemplo, serán S1a, S1b, S1c, y así sucesivamente, e igual con el resto de problemas.

\subsubsection{Espacios de soluciones: descripción y categorización para el análisis}

Antes de la experiencia, uno de los investigadores ha descrito, para cada problema, el espacio de soluciones, si bien, en algunos casos, éste se ha ampliado una vez analizadas las respuestas de los participantes. Una vez recopiladas, para obtener categorías de análisis, las soluciones se han clasificado siguiendo dos criterios:

- Tipo de solución: se han tenido en cuenta los procedimientos matemáticos involucrados en la resolución, se agrupan en la misma categoría las resoluciones 
de un mismo problema que ponen en juego el mismo tipo de razonamiento y procedimientos matemáticos.

- Originalidad: se han clasificado, para cada problema, las resoluciones distinguiendo tres categorías: convencionales/algorítmicas (si involucran procedimientos mecánicos bien conocidos por los estudiantes), poco convencionales (si la resolución pone en juego un razonamiento poco convencional pero que no se sale de lo esperable en función del enunciado del problema), completamente fuera de lo convencional (cuando la resolución se basa en argumentos o procedimientos que no son los esperados).

Para ilustrar la categorización de las soluciones de los problemas, se muestra a continuación, para el problema P1, la descripción detallada de tres posibles soluciones, el esquema de todas las soluciones encontradas y la categorización según el tipo de resolución y según la originalidad.

El problema P1 consistía en demostrar que, en cualquier triángulo rectángulo, la medida de la mediana sobre la hipotenusa es igual a la mitad de la medida de la hipotenusa (véase la Figura 1). A continuación, se presentan tres posibles resoluciones:

S1a) Construcción auxiliar: duplicamos la figura por simetría con eje $\overline{A C}$. Los segmentos $\overline{A M}$ y $\overline{A M^{\prime}}$ unen puntos medios de los lados del triángulo isósceles $C B B^{\prime}$, luego son paralelos a sus lados, de donde $A M C M^{\prime}$ es un paralelogramo, y $\overline{A M}, \overline{C M^{\prime}}$ y $\overline{C M}$ miden lo mismo. Véase la Figura 2.

Figura 2. Primera solución al problema P1

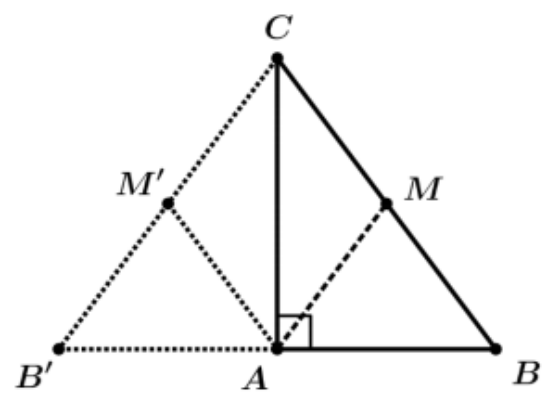

S1j) Geometría analítica: consideramos que el triángulo está apoyado sobre unos ejes coordenados $(A=(0,0), B=(c, 0)$ y $C=(0, b))$. Así, la ecuación de la recta $B C$ es $f(x)=b-\frac{b}{c} x$. Dado un punto $P x=(x, f(x))$ de esa recta, la distancia de $P x$ a $A$ y $B$ es:

$$
\begin{gathered}
d\left(A, P_{x}\right)=\sqrt{x^{2}+\left(b-\frac{b}{c} x\right)^{2}} \\
d\left(P_{x}, B\right)=\sqrt{(c-x)^{2}+\left(-b+\frac{b}{c} x\right)^{2}}
\end{gathered}
$$


De $d(A, P x)=d(P x, B)$, se deduce que $x=\frac{c}{2^{\prime}}$ por lo que el punto que hace que esas distancias sean iguales es $M$.

S1n) El Teorema de Stewart permite calcular lo que mide una ceviana de un triángulo, véase la Figura 3.

Figura 3. Teorema de Stewart (imagen extraída de Wikipedia)

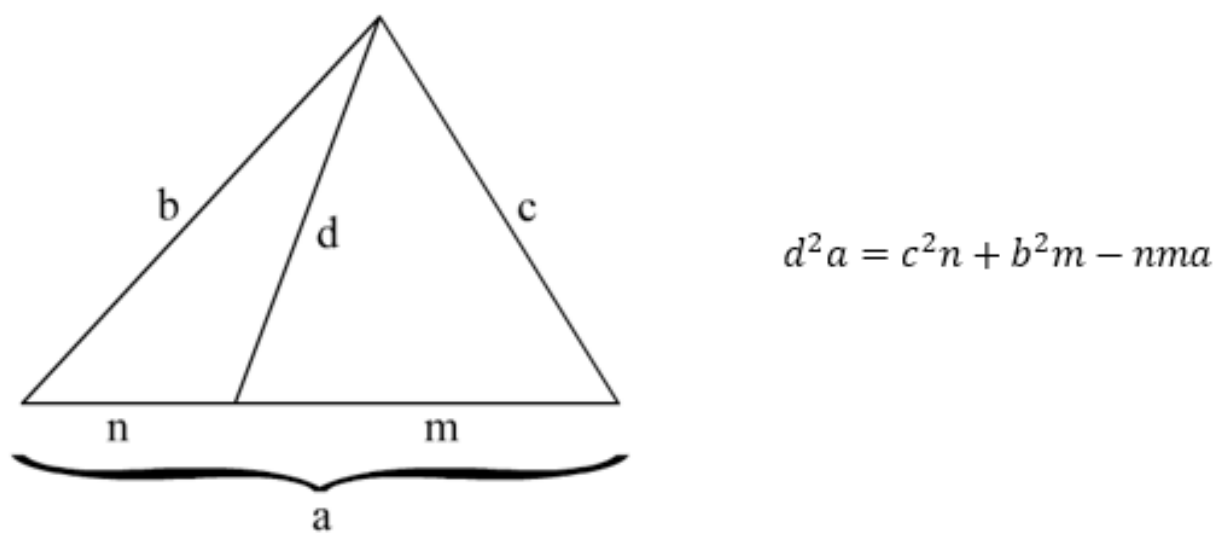

La solución S1a se clasifica como "basada en una construcción auxiliar", y es una solución categorizada como convencional/algorítmica. La solución S1j se clasifica como "basada en argumentos de geometría analítica"; si bien la geometría analítica es común en problemas de este tipo, el uso de puntos genéricos le da complejidad y por eso se clasifica como poco convencional. La solución S1n, encontrada en una de las producciones de los participantes, se basa en el Teorema de Stewart; su aplicación en este problema no es directa ya que no hay datos suficientes, por lo que se clasifica esta solución como no convencional.

Para recoger el espacio de soluciones de cada uno de los problemas, se han resumido las soluciones en una tabla. A continuación, se incluye en la Tabla 1, a modo de ejemplo, el esquema de soluciones del problema P1. Las soluciones en negrita son aquellas que han sido categorizadas como poco convencionales, y las que aparecen, además, subrayadas, han sido categorizadas como no convencionales. Se han señalado con un asterisco las soluciones que no habían sido incluidas inicialmente en el espacio de soluciones.

Tabla 1. Esquema de soluciones del problema 1

\begin{tabular}{|c|c|c|c|c|c|}
\hline S1a & $\begin{array}{c}\text { Construcción auxiliar: } \\
\text { Duplicar por cateto }\end{array}$ & S1f & $\begin{array}{c}\text { Geometría analítica } \\
\text { básica }\end{array}$ & S1k & $\begin{array}{c}\text { Construcción } \\
\text { auxiliar: Mediatriz } \\
\text { de cateto }\end{array}$ \\
\hline S1b & $\begin{array}{c}\text { Construcción auxiliar: } \\
\text { Duplicar por hipotenusa }\end{array}$ & S1g & $\begin{array}{c}\text { Trigonometría: Teorema } \\
\text { coseno }\end{array}$ & $\underline{\text { S1I }}$ & $\begin{array}{c}\text { Rayos de luz y } \\
\text { espejos }\end{array}$ \\
\hline
\end{tabular}




\begin{tabular}{|c|c|c|c|c|c|}
\hline S1c & $\begin{array}{c}\text { Construcción auxiliar: } \\
\text { Circunferencia } \\
\text { circunscrita }\end{array}$ & $\mathbf{S 1 h}$ & $\begin{array}{c}\text { Construcción auxiliar: } \\
\text { Unir puntos medios }\end{array}$ & $\mathbf{S 1 \mathbf { m }}$ & $\begin{array}{c}\text { Trigonometría: } \\
\text { Teorema seno }\end{array}$ \\
\hline S1d & $\begin{array}{c}\text { Construcción auxiliar: } \\
\text { Altura sobre cateto }\end{array}$ & $\underline{\mathbf{S 1 i}}$ & $\begin{array}{c}\text { Construcción auxiliar: } \\
\text { Construir un isósceles }\end{array}$ & $\underline{\mathbf{S 1 n}}$ & Teorema Stewart \\
\hline$\underline{\mathbf{S 1 e}}$ & $\begin{array}{c}\text { Construcción auxiliar: } \\
\text { Duplicar 2 veces por } \\
\text { catetos }\end{array}$ & $\mathbf{S 1 j}$ & $\begin{array}{c}\text { Geometría analítica: } \\
\text { Punto genérico }\end{array}$ & $\underline{\mathbf{S 1} \tilde{\mathbf{n}}^{*}}$ & Arcos capaces \\
\hline
\end{tabular}

\subsubsection{Análisis de las producciones de los estudiantes}

Para dar respuesta a los objetivos, se han analizado los siguientes aspectos en las producciones:

- Fluidez: se ha contado la cantidad de soluciones correctas.

- Flexibilidad: el punto de partida para medir la flexibilidad es la clasificación según el tipo de solución, así, se suman 10 puntos por cada solución que pertenezca a grupos distintos de soluciones. Se suma 1 punto por cada solución que pertenezca a un grupo de soluciones al que ya pertenecía una de las soluciones dadas. Por último, se suman 0,1 puntos por cada solución que es casi idéntica a otra ya dada. Siguiendo a Leikin (2013), una puntuación de 0,1 refleja la falta de razonamiento crítico del estudiante. Esta valoración numérica permite saber cuántas soluciones de cada tipo se han dado: así, si un alumno obtiene 31,2 puntos de flexibilidad, eso nos dice que hay 3 soluciones de grupos distintos, 1 solución de un grupo ya mencionado, y 2 soluciones casi idénticas a soluciones ya dadas.

- Originalidad: el punto de partida es la clasificación de las soluciones según la originalidad y de nuevo, se va a usar un sistema decimal. Se sumarán 10 puntos por cada solución no convencional. Se sumará 1 punto por cada solución caracterizada como poco convencional, basada en modelos o en estrategias aprendidas en otros contextos. Por último, se sumarán 0,1 puntos por cada solución categorizada como convencional/algorítmica.

- Creatividad: siguiendo a Leikin (2013), la creatividad de un resolutor en cada problema se define como el producto de su flexibilidad y su originalidad, y la creatividad total es la suma de los valores de creatividad en cada problema. En efecto, para ser creativo es importante ser flexible y ser original.

- Estilo de razonamiento primario: al tener que hallar más de una solución a un problema con límite de tiempo, la primera solución que se escribe es distintiva. En efecto, puede ser que sea la primera que viene a la mente, o la más corta, o acaso la que da más seguridad al resolutor. En cualquier caso, dado que puede indicar, por ejemplo, si están más cómodos razonando analíticamente o geométricamente, se ha señalado, para cada participante y cada problema, en base a la primera solución hallada, el estilo de razonamiento primario distinguiendo entre algebraico, geométrico o analítico.

- Estilo de razonamiento general: en base a los razonamientos identificados en las soluciones a un mismo problema por parte de un resolutor, se establece un estilo de razonamiento general. 
- Grado de formalidad de las resoluciones: se categoriza distinguiendo tres niveles a partir de todas las soluciones de un mismo resolutor a un mismo problema. Se categorizan como de bajo grado de formalidad aquellas soluciones en las que o bien no se definen los elementos matemáticos utilizados o se definen en referencia a un dibujo. En estas resoluciones se aprecia un bajo nivel de formalización en el razonamiento e, incluso, pueden quedar pasos por probar. En la Figura 4 se muestran las soluciones propuestas por el participante AE6 al problema 1. Se identifican tres soluciones correctas: dos soluciones categorizadas como diferentes y dos categorizadas como casi idénticas. Dos de las soluciones son convencionales (las dos primeras), mientras que la tercera es no convencional. Esta resolución se ha valorado como de bajo grado de formalidad.

Figura 4. Resolución de EA6 al problema P1 categorizada como poco formal

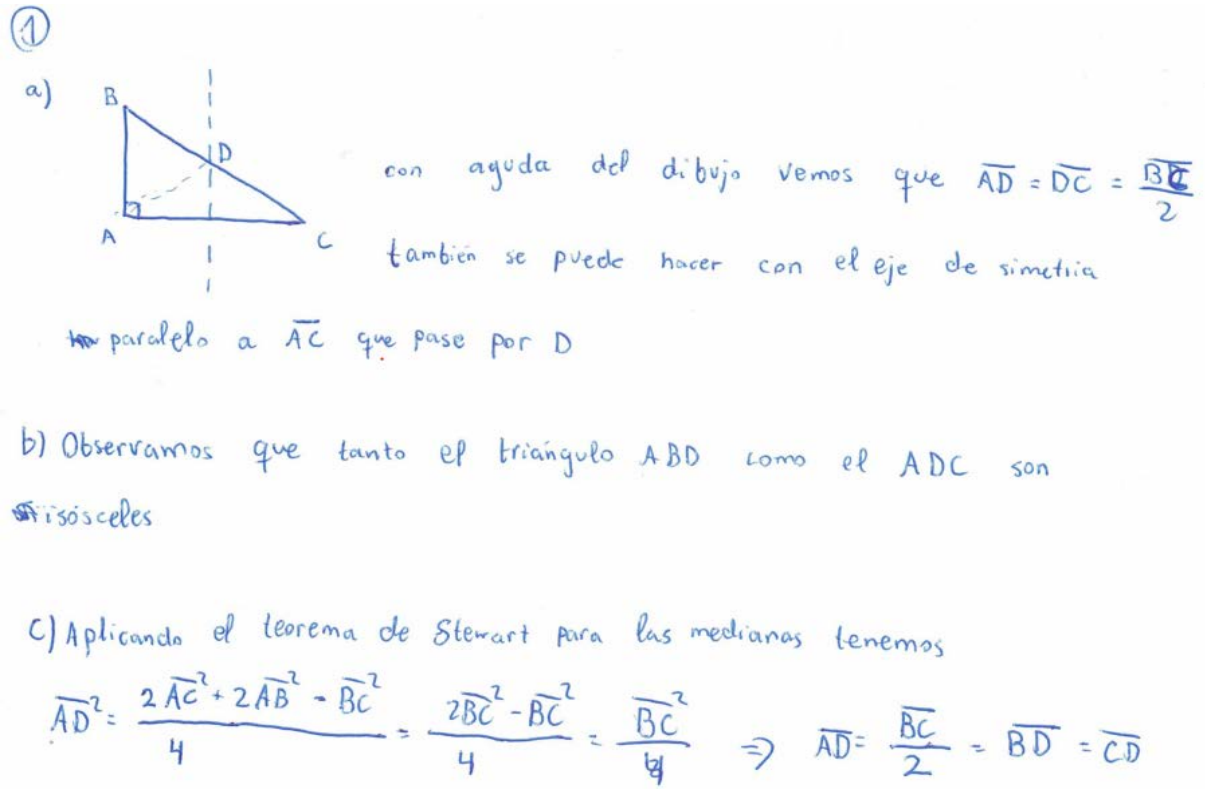

Se categorizan como de grado medio de formalización aquellas resoluciones en las que se identifican razonamientos no del todo formales, a menudo acompañados de esquemas o dibujos. En estas producciones, aunque se aprecian razonamientos deductivos, se obvian algunos detalles. En la resolución del problema $\mathrm{P} 1$ realizada por AM4 (véase Figura 5), se identifican dos resoluciones categorizadas como de una misma categoría, ambas clasificadas como convencionales. Esta resolución ha sido categorizada como de formalidad media.

Finalmente se categorizan como de alto grado de formalidad aquellas resoluciones en las que todos los elementos están bien definidos, se dan todos los detalles del razonamiento, las referencias al dibujo son claras, argumentan bien sus deducciones. En la resolución del problema P1 realizada por AM1 (véase Figura 6), se identifica 
una única solución que se ha categorizado como del alto grado de formalidad ya que se explica y justifica cuidadosamente cada paso del razonamiento.

Figura 5. Resolución de AM4 del problema P1 categorizada como medianamente formal

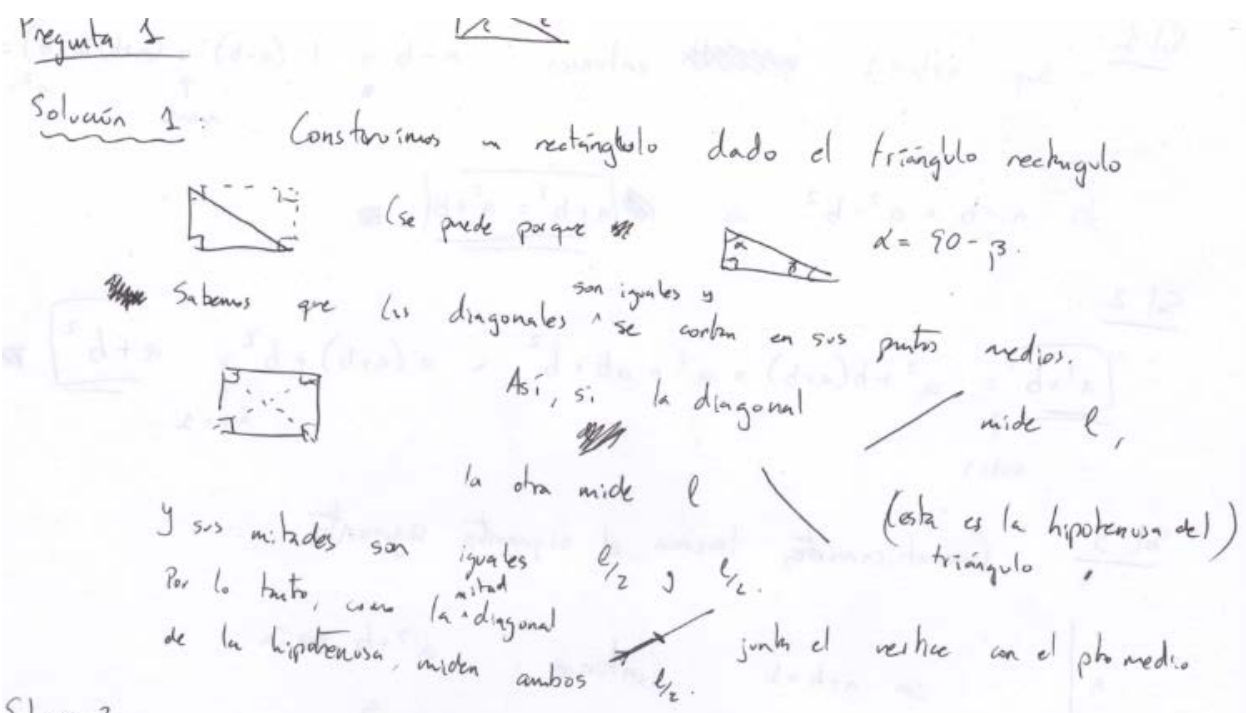

Solouin 2

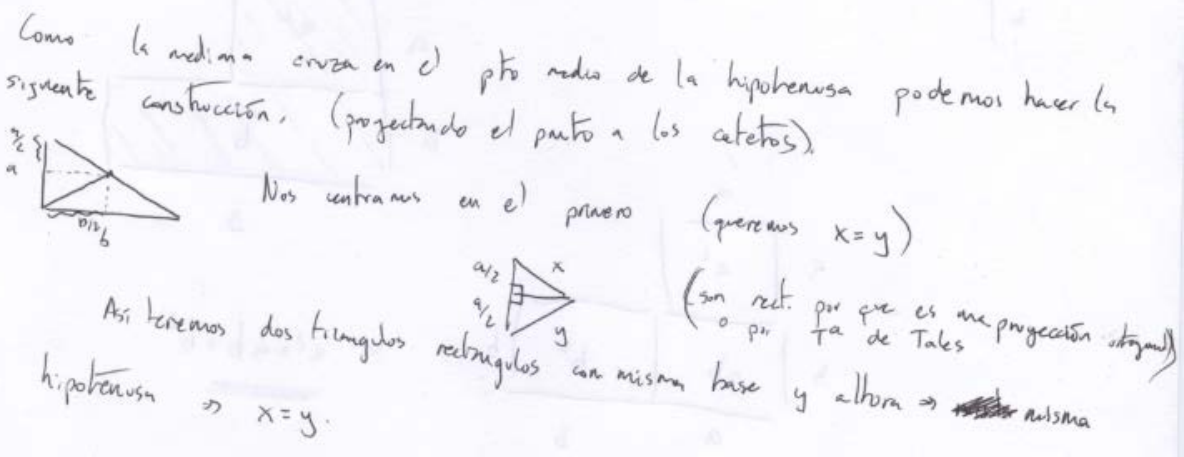

Eficiencia: se mide para cada estudiante en cada problema y se entiende como el uso económico del tiempo y el espacio de trabajo en las resoluciones de los problemas (no escribir cosas innecesarias, buscar soluciones cortas, etc.). Las resoluciones mostradas en la Figura 4 y en la Figura 6 han sido categorizadas como de alta eficiencia, mientras que la de la Figura 5 ha sido categorizada como de eficiencia media.

Una vez descritos en detalle los ocho aspectos considerados en el análisis de las soluciones de los estudiantes a cada problema y cómo se van a valorar, conviene tener en cuenta que se trata de un análisis cualitativo y, como tal, tiene cierta compo- 
nente de subjetividad. En cualquier caso, esto se ha intentado superar con una doble revisión del análisis por parte de los autores con el fin de unificar criterios.

Figura 6. Resolución de AM1 del problema P1 categorizada como altamente formal

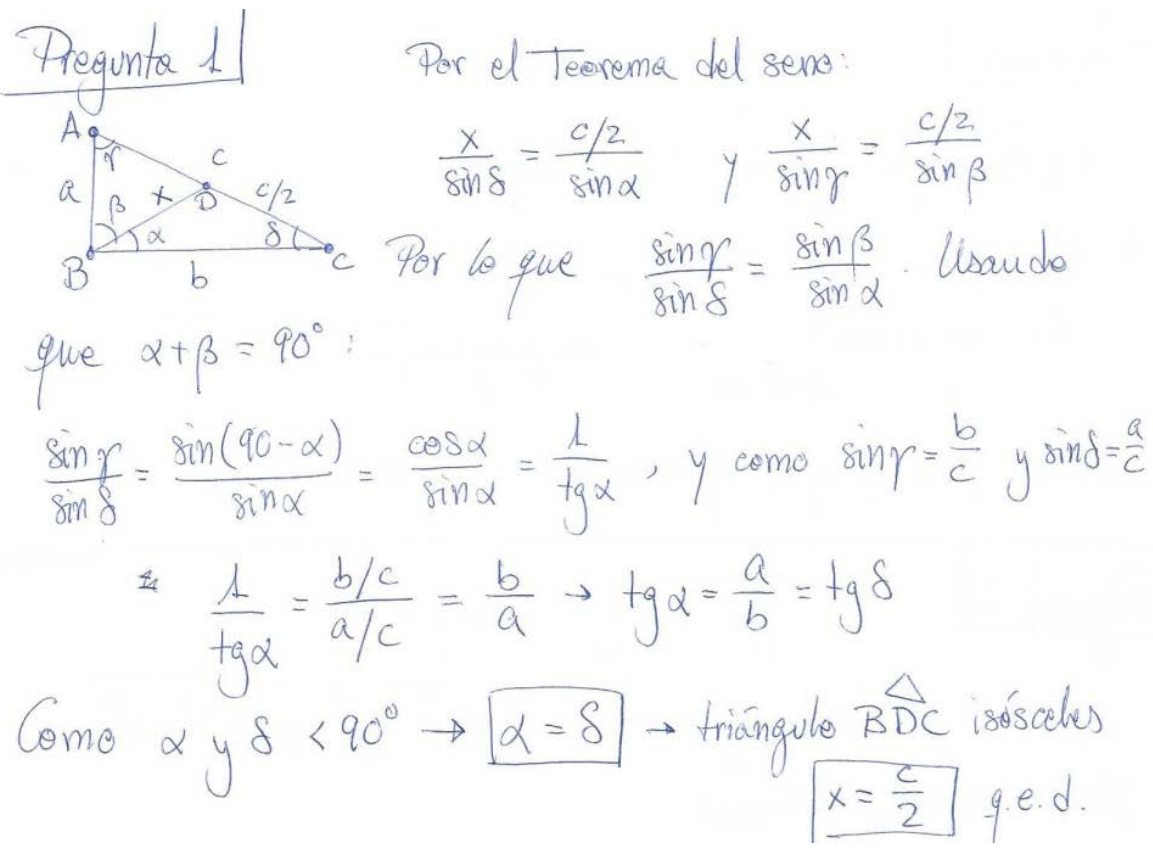

\section{Resultados}

Por limitaciones de espacio no es posible listar en este documento todos los detaIles del análisis. Dado que este estudio ha sido previamente descrito de forma completa en un Trabajo Fin de Máster, remitimos al lector al documento publicado en el repositorio Roderic ${ }^{1}$. A continuación, por las limitaciones de espacio, se presentará únicamente un resumen de los resultados para el problema 1; posteriormente se presentan los resultados globales.

\subsection{Problema 1}

En promedio, los alumnos del máster han aportado 1,6 soluciones por persona, y los de ESTALMAT 2,5 por persona.

En la Tabla 2 se presentan las puntuaciones de flexibilidad, originalidad y creatividad de cada uno de los participantes, usando los criterios descritos en la sección anterior. Para ello, se calculan los valores por solución primero, y después la suma total.

1. El TFM está accesible en el repositorio Roderic a través del siguiente enlace: https://roderic.uv.es/ handle/10550/75764 
Se ha analizado el grado de formalidad de cada estudiante a la hora de escribir, su eficiencia y el estilo de razonamiento (tanto de la primera solución como a nivel global). Los datos se resumen en la Tabla 3.

Tabla 2. Fluidez, flexibilidad, originalidad y creatividad por participante en el problema 1

\begin{tabular}{|c|c|c|c|c|}
\hline Alumno & Fluidez & Flexibilidad & Originalidad & Creatividad \\
\hline AM1 & 1 & 10 & 1 & 10 \\
\hline AM2 & 2 & 20 & 0,2 & 2 \\
\hline AM3 & 3 & 21 & 1,2 & 11,1 \\
\hline AM4 & 2 & 11 & 0,2 & 1,1 \\
\hline AM5 & 0 & 0 & 0 & 0 \\
\hline AE6 & 3 & 20,1 & 10,2 & 101,01 \\
\hline AE7 & 0 & 0 & 0 & 0 \\
\hline AE8 & 5 & 32 & 1,4 & 12,2 \\
\hline AE9 & 2 & 11 & 0,2 & 1,1 \\
\hline AE10 & 4 & 31 & 2,2 & 13 \\
\hline AE11 & 1 & 10 & 10 & 100 \\
\hline
\end{tabular}

Tabla 3. Formalidad, eficiencia y estilo de razonamiento de cada participante en P1

\begin{tabular}{|c|c|c|c|c|}
\hline Alumno & Formalidad & Eficiencia & $\begin{array}{c}\text { Razonamiento } \\
\text { inicial }\end{array}$ & $\begin{array}{c}\text { Razonamiento } \\
\text { predominante }\end{array}$ \\
\hline AM1 & Alta & Alta & Algebraico & Algebraico \\
\hline AM2 & Media & Baja & Geométrico & Algebraico \\
\hline AM3 & Alta & Alta & Geométrico & Geométrico \\
\hline AM4 & Media & Media & Geométrico & Geométrico \\
\hline AM5 & Alta & Baja & Geométrico & Equilibrado \\
\hline AE6 & Baja & Alta & Geométrico & Geométrico \\
\hline AE7 & & & & Equilibrado \\
\hline AE8 & Media & Alta & Geométrico & Geométrico \\
\hline AE9 & Baja & Alta & Geométrico & Algebraico \\
\hline AE10 & Alta & Alta & Geométrico & Geométrico \\
\hline AE11 & Media & Baja & Geométrico & \\
\hline
\end{tabular}

\section{Resultados globales}

Por último, se presentan los resultados globales del análisis de la prueba entera como un global en vez de los ejercicios por separado. En relación a los aspectos ligados a la fluidez, la flexibilidad, la originalidad y la creatividad, lo resultados se muestran en la Tabla 4. 
Para obtener un resumen global del análisis cualitativo relativo a la formalidad, la eficiencia y estilo de razonamiento, se han asignado valores numéricos en las escalas previamente descritas y se han promediado los resultados por problema para cada participante. La Tabla 5 resume estos resultados.

Tabla 4. Fluidez, flexibilidad, originalidad y creatividad por participante en toda la prueba

\begin{tabular}{|c|c|c|c|c|c|c|}
\hline Alumno & Fluidez & Flexibilidad & Originalidad & Creatividad & Posición & $\begin{array}{l}\text { Media de } \\
\text { creatividad }\end{array}$ \\
\hline AM1 & 5 & 41 & 3,2 & 23 & $10^{\circ}$ & \multirow{5}{*}{77,722} \\
\hline AM2 & 7 & 61 & 11,5 & 114,1 & $6^{\circ}$ & \\
\hline AM3 & 10 & 72,1 & 12,7 & 124,21 & $5^{\circ}$ & \\
\hline AM4 & 6 & 51 & 2,4 & 23,1 & $9^{\circ}$ & \\
\hline AM5 & 7 & 43 & 11,5 & 104,2 & $7^{\circ}$ & \\
\hline AE6 & 9 & 70,2 & 21,6 & 214,02 & $4^{\circ}$ & \multirow{6}{*}{205,4} \\
\hline AE7 & 3 & 30 & 10,2 & 102 & $8^{\circ}$ & \\
\hline AE8 & 16 & 115 & 35,8 & 337,3 & $2^{\circ}$ & \\
\hline AE9 & 3 & 21 & 0,3 & 2,1 & $11^{\circ}$ & \\
\hline AE10 & 14 & 122 & 37,4 & 356 & $1^{\circ}$ & \\
\hline AE11 & 5 & 41 & 31,1 & 221 & $3^{\circ}$ & \\
\hline Total & 85 & 667,3 & 177,7 & 1621,03 & & \\
\hline
\end{tabular}

Tabla 5. Grados de formalidad, eficiencia y estilo de razonamiento a lo largo de toda la prueba

\begin{tabular}{|c|c|c|c|c|}
\hline Alumno & Formalidad & Eficiencia & $\begin{array}{c}\text { Razonamiento } \\
\text { inicial }\end{array}$ & $\begin{array}{c}\text { Razonamiento } \\
\text { predominante }\end{array}$ \\
\hline AM1 & Alta & Alta & Algebraico & Algebraico \\
\hline AM2 & Alta & Media & Algebraico & Algebraico \\
\hline AM3 & Alta & Alta & Geométrico & Equilibrado \\
\hline AM4 & Alta & Alta & Equilibrado & Equilibrado \\
\hline AM5 & Alta & Media & Algebraico & Algebraico \\
\hline AE6 & Media & Alta & Algebraico & Algebraico \\
\hline AE7 & Media & Baja & Algebraico & Algebraico \\
\hline AE8 & Media & Alta & Equilibrado & Equilibrado \\
\hline AE9 & Baja & Media & Equilibrado & Equilibrado \\
\hline AE10 & Alta & Alta & Algebraico & Algebraico \\
\hline AE11 & Media & Media & Equilibrado & Equilibrado \\
\hline
\end{tabular}

\subsection{Discusión y conclusiones}

Este estudio pone de manifiesto, pese al reducido número de estudiantes involucrados, una serie de aspectos ya recogidos en estudios previos. Para empezar, refleja 
que tener una formación sólida en matemáticas no implica ser experto en resolución de problemas: alguno de los alumnos de máster con buena formación matemática, no ha conseguido dar una resolución correcta de alguno de los problemas.

Los alumnos del máster por lo general buscan soluciones más algebraicas o analíticas. Si bien también han ofrecido alguna que otra solución más visual-geométrica, tienen bastante interiorizados aspectos de las matemáticas como la trigonometría, las derivadas y la geometría analítica. Es probable que el motivo, o parte, sea que los estudios universitarios de matemáticas se centren precisamente en aspectos muy teóricos, dejando de lado áreas como la geometría euclídea.

La mayoría de los participantes de ESTALMAT, también conocen ramas más teóricas de las matemáticas como el álgebra y el análisis, pero en sus soluciones se aprecia que esas herramientas son, en general, un último recurso. Así como los alumnos del máster han dado soluciones más puramente matemáticas, los alumnos de talento matemático, han intentado encontrar soluciones visuales, menos sofisticadas. Además, han proporcionado con frecuencia soluciones poco comunes, lo cual puede deberse, entre otras razones, a la formación recibida en el proyecto Estalmat o en la participación a sesiones de preparación de olimpiadas matemáticas.

Una de las diferencias que encontramos en las soluciones es el nivel de formalidad a la hora de escribir. Este estudio refleja una realidad esperable: los alumnos del máster son más formales en sus resoluciones. Usan con precisión la notación matemática, sus expresiones matemáticas tienen una buena estructura lógica y nombran las propiedades. Sin embargo, pese al menor grado de formalidad, la escritura matemática de los alumnos ESTALMAT es mucho más formal y correcta que la que suele mostrar un alumno a esas edades.

Otro aspecto estudiado ha sido la eficiencia en la escritura: la hipótesis era que los alumnos de talento matemático serían más eficientes que los del máster en sus resoluciones, y aunque sí que ha habido algún alumno que era muy eficiente (AE8, AE10), la diferencia entre grupos ha sido menor de lo esperado. Esto puede deberse a una de las limitaciones del trabajo, el tamaño reducido de la muestra.

En cuanto a los estilos de razonamiento propuestos por Krutetskii (1976), no hay suficiente información para determinar cómo razonan los alumnos. Es cierto que muchas veces los alumnos del máster daban en primer lugar soluciones algebraicas mientras que los estudiantes de talento las dejaban para el final, pero eso puede ser debido a los problemas elegidos.

Finalmente, se tratará la relación entre la creatividad y el talento matemático, analizando los 4 aspectos fundamentales por separado.

En relación a la fluidez, entre los 5 alumnos del máster han proporcionado una media de 1,75 soluciones por persona en cada problema. En cuanto a los 6 alumnos de ESTALMAT encuestados, han aportado una media de 2,8 soluciones por persona en cada problema. En ese sentido, los alumnos de talento matemático han proporcionado más soluciones a los problemas, aunque hay diferencias notables entre sujetos en ese grupo. El promedio en este grupo ha subido mucho gracias a los alumnos AE8 y AE10, que han aportado considerablemente más soluciones que cualquier otro participante. El grupo del máster, sin embargo, ha sido bastante más uniforme. 
En relación a la flexibilidad, los alumnos del máster han sumado un total de 268,1 puntos, lo que da una media de13,41 puntos por problema por persona. En cuanto a los alumnos con talento matemático, en total han obtenido 399,2 puntos de flexibilidad, lo que da una media de 16,63 puntos por problema por persona. De nuevo, el grupo de alumnos del máster es bastante más uniforme que el de los alumnos de talento. Como ocurría con la fluidez, en ESTALMAT están los tres alumnos que han obtenido las peores puntuaciones (AE7, AE9 y AE11), y los dos alumnos que han obtenido con mucha diferencia las más altas (AE8 y AE10). Una puntuación de 120 puntos en la prueba supone, aproximadamente, dar 3 soluciones notablemente distintas por problema, número que dista mucho de la media calculada antes.

En relación a la originalidad, las puntuaciones de los alumnos del máster suman 41,3 puntos, lo que da una media de 2,07 puntos por persona en cada problema. Eso refleja un nivel no muy alto de originalidad teniendo en cuenta la homogeneidad en este grupo. En cuanto a los alumnos de talento matemático, han obtenido un total de 136,4 puntos, lo que da una media de 5,68 puntos por persona por cada problema. Aquí se empieza a apreciar la diferencia entre talento matemático y formación matemática. Las 4 puntuaciones más altas de originalidad, y con diferencia, han sido de alumnos de ESTALMAT. Hay que tener en cuenta que se trata de un estudio pequeño, y por tanto los resultados son sólo orientativos, pero se aprecia que los alumnos de talento matemático son más versados en la resolución de problemas, tanto en cantidad de soluciones, como en originalidad de las mismas.

Finalmente, en relación a la creatividad, en el máster han obtenido, de media, 15,54 puntos por persona en cada problema. Además, notamos cierta regularidad dentro de la irregularidad: 2 alumnos con poco más de 23 puntos, y tres alumnos con poco más de 100. En cuanto a los alumnos de talento matemático, han recibido de media 51,35 por persona en cada problema. De nuevo, hay heterogeneidad: está en este grupo el alumno con menor puntuación de los 11, con 2,1 puntos, y el alumno con mayor puntuación, con 356 puntos. Destaca que las 4 puntuaciones más altas las han obtenido alumnos de ESTALMAT, y de ellos, hay dos alumnos que han destacado de forma generalizada en todos los problemas: el AE8 y el AE10. En concreto, el alumno AE10 ha destacado más que nadie, habiendo quedado $1^{\circ}$ en 3 de los problemas y $3^{\circ}$ en otro. Ha obtenido mayor nivel de creatividad que el alumno AE8 entregando menos soluciones, lo que indica un mayor nivel de originalidad. Como dato curioso, tanto la puntuación más baja como la más alta las han obtenido los dos alumnos de $4^{\circ}$ de la ESO, uno, el AE9, con talento, pero con un nivel de formación estándar, y el otro, el AE10, con un talento excepcional y una formación curricular y extracurricular bastante completa. Esto da pie a una reflexión: el talento matemático no lo es todo, es necesario complementarlo con una buena formación, porque no es cierto que por tener talento el alumno vaya a aprenderlo todo solo.

El grupo del máster es bastante uniforme en todos los aspectos evaluados, mientras que en el grupo de ESTALMAT es más heterogéneo; esto podría deberse a que la formación matemática es estándar para todos, mientras que el talento es algo innato, distinto en cada individuo, aunque también podría ser un dato casual, puesto que el estudio es pequeño. 
La realización de este estudio ha presentado una serie de limitaciones como el bajo número de participantes, aunque esto ha permitido hacer un análisis minucioso. Por otro lado, al evaluar la originalidad de las respuestas en base al método de Leikin (2013), el análisis se ciñe a las producciones escritas de los estudiantes, omitiendo completo por cualquier información sobre los conocimientos previos de los resolutores. Finalmente, los resultados están limitados por el formato no presencial de la experiencia, que obliga a confiar en que todos han respetado las normas.

\section{AgRADECIMIENTOS}

Los autores de este texto desean agradecer a la profesora Roza Leikin darnos la posibilidad de poner en práctica sus métodos para nuestro experimento y a los 11 participantes voluntarios en la misma. El primer autor está siendo financiado por la beca FPU17/02023 y el proyecto MINECO y FEDER MTM2017-83262-C2-1-P. La segunda autora agradece la ayuda del proyecto de Investigación del Plan Nacional de I+D+I, EDU2017-84377-R financiado MINECO y FEDER. Este trabajo se ha desarrollado en el marco del proyecto del programa propio de Investigación del Vicerrectorado de Investigación de la Universitat de València, convocatoria de Acciones Especiales UV-INV-AE-1557785.

\section{REFERENCIAS BIBLIOGRÁFICAS}

Callejo, M. L. (1994). Un club matemático para la diversidad. España: Narcea.

Davydov, V. V. (1990). Type of generalization in instruction: Soviet studies in mathematics education. Reston, VA, E.E.U.U.: National Council of Teachers of Mathematics.

De Guzmán, M. (s.f.). El tratamiento educativo del talento especial en matemáticas. http://elclubdelamatematica.blogspot.com/2010/06/talento-matematico.html

Díaz, O., Sánchez, T., Pomar, C., Fernández, M. (2008). Talentos matemáticos: Análisis de una muestra. Faisca, 13(15), 30-39.

Jaime, A. y Gutiérrez, A. (2014). La resolución de problemas para la enseñanza a alumnos de educación primaria con altas capacidades matemáticas. En B. Gómez, L. Puig (Eds.), Resolver problemas. Estudios en memoria de Fernando Cerdán (pp. 147-190). Valencia: Universidad de Valencia.

Jaime, A. y Gutiérrez, A. (2017). Investigación sobre estudiantes con alta capacidad matemática. En J. M. Muñoz-Escolano, A. Arnal-Bailera, P. Beltrán-Pellicer, M. L. Callejo y J. Carrillo (Eds.), Investigación en Educación Matemática XXI (pp. 71-89). Zaragoza: SEIEM.

Krutetskii, V. A. (Ed.) (1976). The psychology of mathematical abilities in school children. Chicago, IL, E.E.U.U.: University of Chicago Press.

Leikin, R. (2011). Multiple-solution tasks: from a teacher education course to teacher practice. ZDM - The International Journal on Mathematics Education, 43(6-7), 993-1006.

Leikin, R. (2013). Evaluating mathematical creativity: The interplay between multiplicity and insight. Psychological Test and Assessment Modeling, 55(4), 385-400. 
Leikin, R. y Lev, M. (2007). Multiple solution tasks as a magnifying glass for observation of mathematical creativity. En Woo, J. H., Lew, H. C., Park, K. S. y Seo, D. Y. (Eds.), Proceedings of the 31st Conference of the International Group for the Psychology of Mathematics Education (vol. 3, pp. 161-168). Seúl, Koera: PME.

Miller, R. C. (1990). Discovering mathematical talent (documento ED321487). EE.UU.: ERIC. https://files.eric.ed.gov/fulltext/ED321487.pdf

Miralles, A. (2008). La Experiencia ESTALMAT en la Comunidad Valenciana. ModeIling in Science Education and Learning, 1(5), 39-44.

Polya, G. (1965). Cómo plantear y resolver problemas. México: Trillas.

Shapiro, S. I. (1965). A study of pupil's individual characteristics in processing mathematical information. Voprosy Psikhologii, 2.

Sriraman, B. (2003). Mathematical Giftedness, Problem Solving, and the Ability to Formulate Generalizations: The Problem-Solving Experiences of Four Gifted Students. Journal of Secondary Gifted Education, 14(3), 151-165.

Torrance, E. P. (1974). Torrance tests of creative thinking. Bensenville. IL: Scholastic Testing Service. 